\title{
Wem gehören die Daten? Vertragliche Regelungen, Möglichkeiten und Grenzen bei der Nutzung datenbasierter Produkte
}

\author{
Julia Froese ${ }^{(\bowtie)}$ und Sebastian Straub \\ Institut für Innovation und Technik (iit), Steinplatz 1, 10623 Berlin, \\ Deutschland \\ \{Froese, Straub\} @iit-berlin.de
}

\begin{abstract}
Zusammenfassung. Dieser Beitrag nimmt die Geschäftsbeziehung zwischen Hersteller und Nutzer einer Maschine in den Blick und zeigt auf, welche Aspekte in Bezug auf die Daten, die bei der Nutzung der Maschine entstehen, vertraglich geregelt werden sollten. Aufbauend auf einer Einordnung der Thematik sowie einer kurzen Darstellung der gegenwärtigen (Rechts-)Lage werden anhand eines konkreten Fallbeispiels aus dem Werkzeugmaschinenbau die einzelnen Regelungsgegenstände, Möglichkeiten und Grenzen der Vertragsgestaltung aufgezeigt.
\end{abstract}

Schlüsselwörter: Digitale Souveränität · Datenhoheit · Datenzugriff ·

Nutzungsrechte $\cdot$ Vertragsgestaltung

\section{Einleitung}

Geht es um das rechtliche Schicksal von Daten, die bei oder durch die Nutzung einer Maschine entstehen, so halten sich die gesetzlichen Regelungen hierzu in einem überschaubaren Rahmen. Dies führt zu Unsicherheit auf Seiten der Anwender in Bezug auf die bestehenden Möglichkeiten (so auch Geerenstein 2020) und ist letztendlich einer der Gründe dafür, dass das in der Nutzung der Daten liegende Potenzial nicht voll ausgeschöpft wird. Die bestehenden Regulierungsbestrebungen auf EU-Ebene ${ }^{1}$ zielen daher schon allein aus wirtschaftlichen Gründen darauf ab, diese Situation durch Schaffung eines Rechtsrahmens zu verbessern (Europäische Kommission 2017). Die seit Mai 2019 geltende Verordnung über den freien Verkehr nicht personenbezogener Daten in der EU (VO (EU) 2018/1807) enthält dementsprechend Bestimmungen, die diesbezügliche Hindernisse ${ }^{2}$ beseitigen sollen. Darüber hinaus besteht allerdings weiterhin erheblicher Spielraum, was die Datenhoheit, also die Zuordnung und den Zugriff auf Daten, angeht. Jedoch muss dies nicht zwingend

1 Vgl. für einen Überblick einschließlich einer Einordnung der Mitteilung der EUKommission: Wiebe 2017.

2 Wie etwa Datenlokalisierungsmaßnahmen, die grundsätzlich unzulässig sein sollen, vgl. Art. 4 VO (EU) 2018/1807. 
ein Hemmnis sein, sondern kann durchaus auch Vorteile bringen: Bewegt man sich geschäftlich in einem Bereich, der stark von der Industrie 4.0 geprägt ist oder das Potenzial dazu bietet, und ist man ausreichend über Regelungsbedarf, Ausgestaltungsmöglichkeiten und -grenzen informiert, ist man in der Lage, flexibler und interessengerechter anhand des jeweiligen Einzelfalls zu agieren, als es unter Umständen mit einer gesetzlichen Normierung der Fall wäre.

Anhand eines fiktiven Beispiels einer Geschäftsbeziehung zwischen Hersteller und Nutzer einer Maschine mit Computerized-Numerical-Control (CNC)-Steuerung sollen im vorliegenden Beitrag die Regelungsgegenstände dargestellt werden, die in der Konstellation typischerweise relevant werden, und Hinweise für die vertragliche Ausgestaltung gegeben werden. Eine kurze Übersicht zur Rechtslage soll dazu dienen, den Bedarf vertraglicher Regelungen aufzuzeigen. ${ }^{3}$

Die angesprochene Flexibilität ist beim Thema Datenaustausch/Datensouveränität nach derzeitigem Stand ohnehin geboten: Daten spielen überall eine Rolle, die Digitalisierung zieht sich als Querschnittsthematik durch nahezu alle Rechtsgebiete. Die im folgenden Abschnitt beschriebene bilaterale Beispielkonstellation kann im Grundsatz um beliebig viele Akteure (Plattformbetreiber, weitere Zulieferer etc.) erweitert werden und stellt so gesehen lediglich einen Ausschnitt des durch die Digitalisierung ermöglichten Wertschöpfungsnetzwerks dar, in der sich mit der jeweiligen Konstellation und Branche auch die Interessenlage und damit die Anforderungen an den Vertrag ständig ändern können. Selbst wenn man den Kreis etwas enger zieht und sich etwa, wie hier, auf den Werkzeugmaschinenbau beschränkt, verbleiben eine Vielzahl unterschiedlicher Fallgestaltungen.

Je nachdem können - über diesen Beitrag hinausgehend - dann Themen wie Interoperabilität, die Festlegung (übergreifender) Sicherheitsstandards, die in bestimmten ,vulnerablen' Bereichen, wie beispielsweise Energie und Versorgung, auch gesetzlichen Vorgaben entsprechen müssen, ${ }^{4}$ die Beachtung der Lizenzbedingungen bei der Nutzung von Open-Source-Software, Rechtsfragen grenzüberschreitender Verträge - um nur ein paar Beispiele zu nennen - für die Vertragsgestaltung an Relevanz gewinnen.

\section{Ausgangslage}

Zunächst soll folgendes Beispiel dazu dienen, einen bestehenden Regelungsbedarf aufzuzeigen und die nachfolgenden Ausführungen zu veranschaulichen:

$N$ beliefert Unternehmen verschiedener Industriebranchen mit Bauteilen aus Stahl und Kunststoff, die in größere Maschinen eingesetzt werden. Um die auf die

3 Für eine umfassendere Darstellung siehe Froese/Straub 2020.

4 Im Bereich ,kritischer Infrastrukturen“ sind die Betreiber der jeweiligen Anlagen verpflichtet, bestimmte Sicherheitsstandards einzuhalten und Vorsorgemaßnahmen zu treffen. Im Mai 2021 wurden diese Vorgaben durch das ,IT-Sicherheitsgesetz 2.0` erweitert, beispielsweise um die Pflicht zur Einsetzung von Systemen zur Angriffserkennung, § 8a Abs. 1a Gesetz über das Bundesamt für Sicherheit in der Informationstechnik (BSIG). 
jeweiligen Bedarfe seiner Kunden abgestimmten, unterschiedlichen Teile möglichst effizient herstellen zu können, schließt er einen Vertrag mit $H$, die sich auf die Herstellung von CNC-Fertigungszentren spezialisiert hat. Von $H$ erwirbt $N$ eine automatisierte Universalfräsmaschine, die unterschiedliche Werkstoffe nach verschiedenen Geometrien bearbeiten kann. Die jeweiligen Befehle an die Maschine kann N durch die zugehörige Software einstellen und steuern. Die in der Software gespeicherten Daten will $H$ weiter nutzen, um die Funktionalität der Maschine zu beobachten und, durch einen Vergleich mit den Daten der gleichen Maschine von anderen Nutzern, Verbesserungspotenziale für künftige Versionen der CNC-Fertigungszentren zu identifizieren. Außerdem geben die Daten Aufschluss über den Zustand des Fertigungszentrums. $H$ will diese Daten nutzen und $N$ die Zusatzleistung anbieten, die Maschine nur dann zu reparieren oder Teile auszuwechseln, wenn absehbar ist, dass die Maschine bald nicht mehr die vereinbarte Leistung erbringen kann - also bevor der Reparaturfall überhaupt eintreten würde (Predictive Maintenance). Das ist prinzipiell auch im Interesse von $N$, der jedoch auf der anderen Seite aber auch keine Leistung in Anspruch nehmen möchte, die (noch) nicht erforderlich ist. Die Daten der Maschine sind für $N$ wiederum auch deshalb interessant, weil er so seine eigene Produktion und Produktivität auswerten und seine Prozesse optimieren kann. Ferner möchte N verhindern, dass $H$ durch die Auswertung der Daten seiner Maschine und ihrer Auslastung Kenntnis erlangt über die Auftragslage seines Unternehmens oder sonstige sensible Informationen. $N$ möchte zumindest wissen, welche Daten $H$ zur Verfügung stehen, und Einfluss darauf nehmen können, wie und zu welchem Zweck diese genutzt und womöglich sogar weitergegeben werden.

Ein jeder der Beteiligten kann hier aus unterschiedlichen Gründen ein Interesse daran haben, die Daten, die die Maschine produziert, oder Teile davon, zu nutzen.

Wird hierzu nichts weiter vertraglich vereinbart, löst sich dieses Problem rein technisch: Derjenige, der faktisch auf die Daten zugreifen kann, kann diese für sich nutzen (vgl. Froese/Straub 2020). Daten gehören nach der Rechtsordnung niemandem: Es gibt kein Eigentum oder ein vergleichbares ausschließliches Hoheitsrecht an Daten. Dies gilt auch für personenbezogene Daten. Die strengen Vorgaben des Datenschutzes und die vergleichsweise weitgehenden Ansprüche der betroffenen Person liegen darin begründet, dass die erfassten Daten das allgemeine Persönlichkeitsrecht und damit einen grundrechtssensiblen Bereich betreffen, der besondere Anforderungen an die Rechtmäßigkeit einzelner Eingriffe stellt (BVerfGE 1983). Ein Eigentum an Daten ist damit nicht verbunden.

Neben dem Datenschutzrecht gibt es in anderen Teilrechtsgebieten vereinzelt Verfügungsrechte an Daten und punktuelle Schutzrechte, wie etwa im Urheberrecht, welches in $\S \S 4$ und $87 \mathrm{ff}$. UrhG der Person, die eine Datenbank erstellt hat, unter bestimmten Voraussetzungen gewisse Rechte gewährt. ${ }^{5}$ Hier sind Einzeldaten mittelbar geschützt, über den Schutz der Datenbank als solcher sowie den Schutz der Investition in Aufbau und Pflege der Datenbank. Handelt es sich bei den in Daten verkörperten Informationen um Geschäftsgeheimnisse, sind diese durch das Geschäftsgeheimnisgesetz (vgl. $\S \S 6$ ff. GeschGehG) vor unberechtigter Erlangung, Nutzung

5 S. hierzu im Einzelnen Froese/Straub 2020. 
und Offenlegung geschützt. Während auf Rechtsfolgenseite durchaus weitreichende Ansprüche bestehen, liegt die Schwierigkeit für die Geschädigten hier eher auf der Tatbestandsseite, nämlich bei der Frage, ob es sich bei einer Information um ein Geschäftsgeheimnis im Sinne des $\S 1$ GeschGehG handelt. Um diesen Nachweis zu erbringen, muss das betroffene Unternehmen unter anderem darlegen können, dass angemessene Geheimhaltungsmaßnahmen zum Schutz der Information ergriffen worden sind. Hierzu müssen die jeweiligen Informationen dergestalt klassifiziert werden, dass bei steigender Bewertung der Schutzbedürftigkeit entsprechend risikoadäquate Maßnahmen technischer, organisatorischer und rechtlicher Art ergriffen worden sind (Froese/Straub 2020).

Da die genannten Regelungen lediglich punktuellen Schutz bieten und die Schutznormen nur einen eng begrenzten Anwendungsbereich haben, sind vertragliche Absprachen unerlässlich, um einen sachgerechten Ausgleich der jeweiligen Interessen auch im Einzelfall herzustellen.

Allgemeine Obergrenzen werden ferner durch zwingende Regelungen im Zivilrecht $(\S \S 138,242,305$ ff. BGB) oder auch durch das Wettbewerbsrecht ( $\S 18$, 19 GWB) festgelegt. Jedoch sollen diese Normen lediglich krasse Missverhältnisse verhindern und darüber hinaus die grundsätzlich bestehende Vertragsfreiheit nicht beschränken (siehe hierzu auch unter 4.3). Das hat zur Folge, dass die meisten benachteiligenden Regelungen nicht die Schwelle zur Unwirksamkeit überschreiten und man daher als Vertragspartner selbst gehalten ist, Standards zu setzen.

\section{Ausgestaltungsmöglichkeiten und Hinweise}

Einleitend sollen die folgenden Ausführungen den Inhalt dieses Abschnitts erläutern.

Die Vertragsparteien können Regelungen zu Daten in einen bestehenden Vertrag aufnehmen (etwa in einen Kauf- oder Wartungsvertrag) oder in einem eigenen Vertrag regeln (Hoeren/Uphues 2020) - allgemeine, vertragliche Aspekte spielen in beiden Fällen eine Rolle. Diese werden daher im Folgenden ebenfalls dargestellt, allerdings nur insoweit vertieft, wie es dem Fokus dieses Beitrags entspricht. Auf weiterführende Informationen wird jeweils in den Fußnoten hingewiesen.

Ferner sind bei Vertragsverhandlungen verschiedene Punkte zu beachten, die nicht direkt sichtbar im Vertrag auftauchen, aber bedeutend für seine Gültigkeit, seine Beständigkeit und seinen Nutzen sind. Dies ist zum einen die Frage der Vertragsart (siehe hierzu 3.1), zum anderen die Beachtung zwingenden Rechts und allgemeiner Rechtsgrundsätze wie Normenklarheit und -bestimmtheit. Wenngleich nicht jede Missachtung oder jeder kleine Fehler Konsequenzen nach sich zieht oder gar zu einer Nichtigkeit des gesamten Vertragswerks führt, sollte eine Vereinbarung doch abseits der rein technischen Wirksamkeit Vorteile für eine bestehende Zusammenarbeit bringen und diese erleichtern, und nicht verkomplizieren. Die wichtigsten Fragen in diesem Zusammenhang werden daher unter 3.3 adressiert. 


\subsection{Vertragsart}

Das Bürgerliche Gesetzbuch (BGB) enthält besondere Bestimmungen für einzelne Vertragsarten, die über diejenigen des allgemeinen Schuldrechts hinausgehen. Diese orientieren sich an klassischen ,Geschäftstypen“ wie Kauf, Pacht, Miete etc. und erfassen Rechtsgeschäfte mit Daten, wenn überhaupt, nur mittelbar. Bei einer atypischen Konstellation wie der vorliegenden kann man entweder mehrere Vertragstypen kombinieren und die jeweils passenden Elemente aus einem Teilrechtsgebiet wählen oder man schafft einen eigenständigen, von den geregelten Typen losgelösten Vertrag sui generis. Dies ist für die Frage relevant, welche Rechtsvorschriften zur Bewertung des Vertrags und zur Lösung etwaiger Meinungsverschiedenheiten herangezogen werden. Bei einem typengemischten Vertrag sind dies primär Spezialregelungen. ${ }^{6}$ In der vorliegenden Konstellation wird es auf einen Vertrag sui generis hinauslaufen, mit der Folge, dass das allgemeine Schuldrecht zur Anwendung kommt (Emmerich 2019).

\subsection{Vertragsbestandteile}

Zwingende, wesentliche Vertragsbestandteile sowie weitere, den Interessen der Parteien dienende Gesichtspunkte zusammengenommen, kommt man auf folgende Regelungsgegenstände, die in diesem Abschnitt im Einzelnen dargestellt werden: Vertragsparteien, Vertragsgegenstand, Leistungspflichten/Zugriffsrechte/Nutzungszwecke, Beschaffenheit des Vertragsgegenstands, Bestimmungen technischer Art zur Übertragung und Bereitstellung der Daten, Laufzeit des Vertrags und Kündigung, Haftung für durch fehlerhafte Daten entstandene Schäden, Fragen zur Durchsetzung des Vertrags.

Zusätzlich sollten in einer Präambel oder an anderer Stelle weitere Erläuterungen angefügt werden, die nicht primär die vertraglichen Leistungen betreffen, sondern den Inhalt, den zugrunde liegenden Sachverhalt und Regelungszweck des Vertrages näher beschreiben. Bei sehr technisch gelagerten oder komplizierten Sachverhalten dient dies dem besseren Verständnis außenstehender Nutzer und verschafft Klarheit bei Auslegungsschwierigkeiten. Die Erläuterungen umfassen idealerweise Definitionen der genutzten Begriffe, Beschreibungen der technischen Umsetzung und der genutzten Systeme sowie eventuell kurze Ausführungen zum Hintergrund des Vertrags (Kuß 2020).

Vertragsparteien. Die Nennung der Vertragsparteien ist essenzieller Bestandteil eines wirksamen Vertrages. Handelt es sich bei einer der Parteien um einen Konzern, ist darauf zu achten, dass exakt die Gesellschaft bezeichnet wird, mit der der Vertrag geschlossen werden soll. Gesellschaften müssen außerdem wirksam vertreten werden ${ }^{7}$

\footnotetext{
${ }^{6}$ Wobei allerdings umstritten ist, nach welcher Methode die einschlägigen Regelungen bestimmt werden (Emmerich 2019).

7 Die Anforderungen an eine wirksame Vertretung sind je nach Gesellschaftsform unterschiedlich, vgl. zum Beispiel § 125 HGB für die offene Handelsgesellschaft (oHG) oder $§ 35$ GmbhG für die Gesellschaft mit beschränkter Haftung $(\mathrm{GmbH})$.
} 
Vertragsgegenstand. Der Vertragsgegenstand muss bezeichnet werden. Bei Daten ist dies aufgrund ihrer fehlenden Verkörperung herausfordernd - eine möglichst detaillierte Aufzählung und Einordnung aber gerade deshalb wichtig. Damit es im Nachgang nicht zu Regelungslücken kommt, sollte darauf geachtet werden, die anfallenden Daten umfassend einzubeziehen. Unter Umständen bietet es sich hier an, nach Nennung konkreter Daten(-gruppen) eine ,Auffangformulierung' zu ergänzen, die sich auf, alle weiteren Daten' bezieht, die, durch oder bei Betrieb von [XY] entstehen'

Sollen außerdem für verschiedene Arten von Daten verschiedene Rechte vorgesehen werden, sollte an dieser Stelle die entsprechende Gruppierung und Zuordnung erfolgen. In den folgenden vertraglichen Ausführungen können dann die jeweiligen Gruppenbezeichnungen verwendet werden. Anhand welcher Merkmale die Zuordnung erfolgt, kommt auf die Interessenlage im Einzelfall an. Möglich ist etwa eine Unterscheidung nach dem situativen Entstehungskontext (Bsp.: Fehlermeldungen) oder eine Unterscheidung von maschinenbezogenen und produktbezogenen Datensätzen (Geerenstein 2020). Je nach Menge und Komplexität bietet es sich an, die Daten in einem separaten Dokument im Anhang zu listen und im Vertrag selbst nur darauf zu verweisen.

An dieser Stelle sollten zudem der Datenspeicherort bezeichnet werden sowie Möglichkeiten und etwaige Erfordernisse, hierauf zuzugreifen.

Leistungspflichten, Zugriffsrechte und Nutzungszwecke. Die Bestimmungen zu Zugriffs- und Verfügungsrechten bilden den zentralen Kern einer Vereinbarung über Daten einer Maschine. Bei der Entscheidung darüber, welcher Partei man in welchem Umfang welche Rechte einräumen möchte, können der bestehende Gestaltungsspielraum voll ausgeschöpft und die konkreten Umstände des Einzelfalls optimal berücksichtigt werden. Den Parteien steht es frei, wie detailliert sie hier werden wollen. Im Interesse der Verständlichkeit sowie zur Minimierung möglicher Fehlerquellen ist es jedoch ratsam, von kleinteiligen Regelungen abzusehen, wenn sie nicht zur Wahrung der Interessen notwendig sind. Auch hier ist es wichtig, zu prüfen, ob man eine den Sachverhalt erschöpfende Regelung getroffen hat und nicht durch die gewählten Formulierungen bestimmte Konstellationen später ,durch das Raster fallen'. Sicherstellen kann man das (wie oben), indem man eine umfassende, sich auf alles beziehende Regel aufstellt, die man , vor die Klammer zieht' und die immer dann gilt, sofern keine der besonderen Regelungen einschlägig ist

Verdeutlichen lässt sich dies am ehesten an einem Beispiel: Daten lassen sich einsehen, man kann sie speichern, kopieren, mit anderen Daten in Relation setzen, zusammenfassen, auswerten, weitergeben, veröffentlichen etc. Diese Begriffe sind weder klar definiert noch trennscharf voneinander abzugrenzen, es kann also zu Lücken oder Widersprüchen kommen, wenn man etwa formulieren würde: ,A darf die Daten X auswerten, B soll einen lesenden Zugriff auf die Daten Y erhalten'. Darf B die Daten auch speichern oder nicht? Ist darüber hinaus jeder weitere Zugriff auf die Daten Y verboten? Wenn A für die Auswertung der Daten X zwangsläufig auch die Daten Y braucht, darf er dann auch auf diese zugreifen, auch wenn dies nicht ausdrücklich vorgesehen wurde? Durch künftige Ereignisse können zudem weitere 
Fragen entstehen, die man bei Vertragsschluss noch nicht bedacht hatte. Wichtig ist hier also:

- Zunächst müssen sich die Parteien auf gemeinsame Begriffe und ihre Bedeutung verständigen und dies (etwa in der Präambel, s. o.) schriftlich festhalten. Hierbei kann man die einzelnen Rechte, die in der konkreten Konstellation sinnvollerweise zusammenhängen (müssen), zusammenfassen. Beispielsweise könnte eine

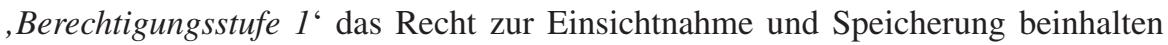
und weitergehende Befugnisse wie Vervielfältigung, Weitergabe und jegliche Art der Verarbeitung ausschließen; eine ,Berechtigungsstufe 2' neben den Rechten aus Stufe 1 noch die Rechte zur Vervielfältigung und Verarbeitung, aber kein Recht zur Weitergabe an Dritte beinhalten, usw.

- Anschließend erfolgt eine Zuordnung der gewählten Befugnisse zu den Daten einerseits und zu den Personen andererseits. Man kann eine Person bestimmen, der grundsätzlich alle Rechte zustehen und den anderen Beteiligten die jeweils passenden Zugriffsrechte zu bestimmten Zwecken einräumen. Diese Zuordnungen können auch für jede Datengruppe getrennt erfolgen.

Im Eingangsbeispiel benötigt $\mathrm{H}$ Daten über den Zustand der einzelnen Werkzeuge und Systeme (Daten ,A'), um einerseits Optimierungsmöglichkeiten für künftige Fertigungszentren und andererseits einen sich anbahnenden Reparaturbedarf zu identifizieren. $\mathrm{H}$ muss diese Daten also auswerten und braucht entsprechende Rechte zur Einsichtnahme und Verarbeitung. Für $\mathrm{N}$ ist es wichtig, dass transparent ist, welche Daten H nutzt, insbesondere, wenn es darum geht, hierauf aufbauend weitere Leistungen zu verkaufen. Zusätzlich möchte N die Daten zu Laufzeit und Auslastung der Maschine auswerten (Daten ,B`), um seine Produktivität und seine Prozesse zu optimieren. Da diese Daten mittelbar Aufschluss über die Auftragslage von N geben, soll H hierauf nicht - auch nicht lesend - zugreifen können. Dieser Interessenlage entspricht es am ehesten, $\mathrm{N}$ einen lesenden und $\mathrm{H}$ einen weitergehenden Zugriff auf die Daten A zuzuweisen, während die Daten B nur N zugänglich sein werden.

Die Nennung der jeweiligen Nutzungszwecke kann in manchen Konstellationen hilfreich sein, um spätere Auslegungsschwierigkeiten etwa bei Gewährleistungsansprüchen zu vermeiden (siehe auch ,Beschaffenheitsvereinbarung').

Möglich, aber nicht zwingend, ist es, dies um eine ausdrückliche Aussage zum Verbot zur Weitergabe der Daten an Dritte zu ergänzen. Wenn Daten zu bestimmten Zwecken an Dritte weitergegeben werden sollen (was aus unterschiedlichen Gründen im Interesse der Parteien sein $\mathrm{kann}^{8}$ ), sollten die entsprechenden Nutzungsbefugnisse auf die im Einzelnen festgelegten Zwecke beschränkt werden und jegliche Weitergabe oder sonstige weitere Verwendung ausgeschlossen werden, damit sichergestellt ist, dass keine Geschäftsgeheimnisse an Unbefugte weitergegeben oder sonstige geschäftsschädigende Handlungen vorgenommen werden.

\footnotetext{
8 Etwa: Veräußerung von Daten als Geschäftsmodell, Einbindung Dritter für weitere Auswertungen im eigenen Interesse oder auch eine grundsätzliche Trennung von Maschine und Daten etwa durch bestehende Kooperationsmodelle zwischen Maschinenherstellern und Datenplattformen, um nur ein paar mögliche Konstellationen der Erweiterung zu nennen.
} 
Beschaffenheitsvereinbarung. In Konstellationen, in denen auf Grundlage der Daten, die durch eine Partei überlassen werden, weitere Dienste oder Produkte angeboten werden, empfiehlt es sich, hinsichtlich der Beschaffenheit der Daten eine Vereinbarung zu treffen. Im Rahmen einer solchen Beschaffenheitsvereinbarung können positive oder negative Leistungsstandards festgelegt werden (Kuß 2020). Positive Leistungsstandards können etwa Anforderungen hinsichtlich der Datenqualität, des Datenformats oder der Aktualität der Daten enthalten. Die Vertragsparteien konkretisieren im Rahmen der Beschaffenheitsvereinbarung die vertraglich geschuldete Leistungspflicht und bestimmen damit zugleich die sog. Sollbeschaffenheit der Daten. Durch die Festlegung von Qualitätseigenschaften stellt der Leistungsempfänger sicher, dass die Daten für den vorgesehenen Zweck (z. B. ein Zustands- und Wartungsmonitoring) verwendet werden können. Zudem sorgt eine Beschaffenheitsvereinbarung für mehr Rechtssicherheit. Denn anhand von konkret festgelegten Kriterien lassen sich negative Abweichungen in der Datenqualität leichter feststellen. Wird die zugesicherte Datenqualität unterschritten, liegt ein Mangel vor und auf Seiten des Leistungsempfängers können Gewährleistungs- und/oder Schadensersatzansprüche entstehen. Legen die Vertragsparteien keine Sollbeschaffenheit fest, lässt sich die Mangelfreiheit der Daten deutlich schwerer ermitteln. In diesem Fall kommt es in Bezug auf die Mangelfreiheit darauf an, ob sich die bereitgestellten Daten für den im Vertrag vorgesehenen Zweck eignen. Liegt der Zweck der Datenbereitstellung beispielsweise in der Erbringung einer Dienstleistung und erweisen sich die Daten als hierfür ungeeignet, stellt dies einen Mangel dar. Regelt der Vertrag weder eine Sollbeschaffenheit noch einen erkennbaren Verwendungszweck, kommt es für die Mangelfreiheit auf die Geeignetheit zur gewöhnlichen Verwendung an. Es ist dann zu prüfen, ob die Daten eine Beschaffenheit aufweisen, die bei Gütern der gleichen Art üblich sind. Die Herbeiziehung eines entsprechenden Vergleichsstandards stellt sich in Bezug auf Daten häufig als schwierig dar. Aus diesem Grund ist eine möglichst konkrete Beschaffenheitsbeschreibung hilfreich, Unklarheiten in Bezug auf die geschuldete Leistung auszuräumen. Daneben sorgt auch die Festlegung eines konkreten Verwendungszwecks für mehr Rechtssicherheit

\section{Bestimmungen technischer Art zur Übertragung und Bereitstellung der Daten.}

Neben einer vertraglichen Regelung zur Datenbeschaffenheit sollten die Vertragsparteien zudem die Umstände der Datenbereitstellung festgelegen. Das betrifft insbesondere die technische Seite. $\mathrm{Zu}$ bestimmen sind unter anderem die zu verwendenden Schnittstellen oder Datenformate. Darüber hinaus sollte eine Vereinbarung darüber getroffen werden, in welchem zeitlichem Abstand Daten geliefert werden und wer die Kosten für die Anbindung an Kommunikationsdienstleister zu tragen hat (Sattler 2020). Da das Verfahren zur Übergabe der Datensätze einen besonders erfolgskritischen Faktor darstellt, sollten die technischen Belange zuvor mit den ITAbteilungen abgestimmt werden (Apel 2021)

Laufzeit des Vertrags und Kündigung. Verträge in der vorigen Konstellation werden sich wahrscheinlich nicht in einem einmaligen Leistungsaustausch erschöpfen: Die Maschine produziert fortwährend Daten, die sukzessive entstehen und wahrscheinlich auch so genutzt werden sollen. Es wird sich also in den meisten Fällen um ein sog. Dauerschuldverhältnis handeln (vgl. Gaier 2019). Da dieses also auch nach dem ersten Leistungsaustausch fortbesteht, können sich die Parteien 
an dieser Stelle überlegen, welche Ausgestaltung der Laufzeit am besten zu ihrer Geschäftsbeziehung passt: Schließt man einen Vertrag auf unbestimmte Zeit, kann dieser durch ordentliche Kündigung (für die Zukunft) beendet werden (Gaier 2019). In diesem Fall sind gleichzeitig Bestimmungen für die einzuhaltende Frist und die Modalitäten der Kündigung aufzunehmen. Soll der Vertrag befristet sein, endet er nicht durch Kündigung, sondern durch Zeitablauf. Das Ende eines befristeten Vertrags kann dabei von einem Zeitpunkt oder auch dem Eintritt eines Ereignisses abhängig gemacht werden. Sowohl befristete als auch unbefristete Verträge können gemäß 314 BGB durch außerordentliche Kündigung ${ }^{9}$ beendet werden, wenn eine Fortsetzung des Vertrags ansonsten unzumutbar wäre ${ }^{10}$

An dieser Stelle muss auch eine Regelung dazu getroffen werden, was mit den bis dato entstandenen Daten nach Vertragsende passiert. Im Beispielfall sind beide Parteien nach der getroffenen Vereinbarung sowieso schon im Besitz der für sie wichtigen Daten. Hier sollte man Aussagen dazu treffen, dass die entsprechenden Auflagen und Beschränkungen auch nach Vertragsende fortbestehen. Relevanter ist eine Regelung in Fällen, in denen nur eine Partei über die Daten der Maschine verfügen kann - hier könnte es zu Konstellationen kommen, in denen die jeweils andere Partei mittelbar an den Vertrag gebunden ist, da durch Weigerung der Herausgabe bestimmter Daten bei Kündigung ein Vertragspartnerwechsel verhindert wird (vgl. auch Vogel 2020).

Haftung für durch fehlerhafte Daten entstandene Schäden. Gerade bei der maschinellen Fertigung von Bauteilen entscheiden häufig Kleinigkeiten über die Art und Güte eines Werkstücks. Bereits kleinste Abweichungen können zu Fehlern führen, die die weiterveräußerten Produkte unbrauchbar machen oder im schlimmsten Fall auch Schäden beim Kunden bis hin zur Betriebsstilllegung nach sich ziehen können. Gehen die hervorgerufenen Schäden auf fehlerhafte Daten zurück, stellt sich die Frage, inwieweit der Bereitsteller der Daten hierfür einzustehen hat - oder anders ausgedrückt - inwieweit er für Datenfehler haftet

Ein Anspruch auf Schadensersatz im Rahmen eines Vertragsverhältnisses setzt regelmäßig voraus, dass eine Leistungspflicht verletzt wird. Zur Feststellung, ob eine Vertragspartei eine Leistungspflicht verletzt hat, ist es notwendig, die vertraglich geschuldeten Leistungen möglichst genau $\mathrm{zu}$ bezeichnen. Dabei sollte auch festgehalten werden, welche Qualität konkret geschuldet wird (Kuß 2020). Schadensersatzpflichtig wird eine Vertragspartei jedoch nur, wenn sie die Schlechtleistung zu vertreten hat. Dabei unterscheidet das BGB in § 276 Abs. 1 BGB zwischen Vorsatz und Fahrlässigkeit. Im Bereich der Fahrlässigkeit wird zudem zwischen einfacher Fahrlässigkeit und grober Fahrlässigkeit unterschieden. Verpflichtet sich

9 Bei einer außerordentlichen Kündigung müssen die regulären Fristen (d. h. die festgelegte ordentliche Kündigungsfrist oder das Ende der Vertragslaufzeit) nicht eingehalten werden. Je nach den Umständen ist aber noch eine entsprechend angemessene Frist abzuwarten, bis der Vertrag beendet ist (vgl. § 314 Abs. 2 und 3 BGB).

10 Was unzumutbar ist, entscheidet sich im Einzelfall unter Beachtung der beiderseitigen Interessen. Je klarer für Außenstehende Hintergründe und Zwecke des Vertrags sind, desto interessengerechter lassen sich unbestimmte Rechtsbegriffe wie ,Unzumutbarkeit“ auslegen. 
eine Vertragspartei zur Bereitstellung von Daten, kann sie also auf Schadensersatz in Anspruch genommen werden, wenn sich die Daten als fehlerhaft erweisen (zum Mangelbegriff siehe oben) und sie diesen Umstand zu vertreten hat. Dieses im Gesetz vorgesehene Verschuldensprinzip stößt jedoch in der Praxis häufig an seine Grenzen, denn der zu beweisende Ursachenzusammenhang zwischen fehlerhafter Datenquelle und dem eingetretenen Schadensereignis lässt sich im Nachhinein häufig nicht nachvollziehen. Aufgrund dieser in vernetzten Umgebungen häufig anzutreffenden Intransparenz macht es Sinn, Beweiserleichterungen vertraglich vorzusehen, damit etwaige Ansprüche leichter durchgesetzt werden können (Kuß 2020).

Daneben haben die Vertragsparteien regelmäßig ein Interesse, die Haftung, die zumeist auf einen finanziellen Ausgleich in Form von Schadensersatz gerichtet ist, auszuschließen. Dabei steht den Vertragsparteien aufgrund der im Zivilrecht bestehenden Vertragsfreiheit ein hoher Handlungsspielraum zur Verfügung. Handelt es sich um individuell ausgehandelte Verträge (im Gegensatz zu Allgemeinen Geschäftsbedingungen, die sich an eine Vielzahl von Vertragspartnern richten), kann die Haftung nahezu vollständig ausgeschlossen werden. Ausgenommen ist die Haftung für vorsätzlich hervorgerufene Schäden (§ 276 Abs. 3 BGB) oder Fälle, in denen ein Gesetz anderweitig einen Ausschluss der Haftung verbietet (z. B. die Haftung für Schäden nach dem Produkthaftungsgesetz).

Soll die Haftung im Rahmen von Allgemeinen Geschäftsbedingungen (AGB) ausgeschlossen werden, greifen strengere gesetzliche Vorgaben, die insbesondere Haftungsausschlüsse bei Verletzung von Leben, Körper, Gesundheit und bei grobem Verschulden untersagen. Diese im AGB-Recht zwingend anzuwendenden Vorgaben finden bei Individualvereinbarungen zwar keine Anwendung. Dennoch finden sich in vielen Verträgen Haftungsklauseln, die sich am AGB-Recht orientieren.

Zudem wird in der Praxis die Haftung häufig höhenmäßig begrenzt und beschränkt sich auf Schäden, die bei Vertragsschluss vorhersehbar sind. Nicht möglich ist es in diesem Zusammenhang, sich vertraglich von sog. ,Kardinalspflichten“ zu lösen. Dabei handelt es sich um Leistungspflichten, deren Verletzung den Vertragszweck gefährden würde und auf deren Erfüllung der Vertragspartner berechtigterweise vertrauen darf. Ist beispielsweise die technische Übermittlung von Daten über eine zuvor definierte Schnittstelle vereinbart, stellt dies eine wesentliche Leistungspflicht dar. Die Haftung für die Verletzung dieser Leistungspflicht darf so dann nicht beschränkt werden. Wer eine derartige Pflicht vorsätzlich oder fahrlässig verletzt, haftet für den dadurch entstandenen Schaden.

Fragen zur Durchsetzung des Vertrags. Es gibt Verschiedenes, was man beachten kann, um die Einhaltung der vertraglichen Bestimmungen abzusichern. Jeder vertragliche Anspruch ist, wenn er nicht erfüllt wird, gerichtlich einklagbar. An dieser Stelle sei bereits auf die Wichtigkeit nachvollziehbarer, verständlicher Regelungen hingewiesen: Ein Anspruch muss von den Gerichten auch als solcher erkannt werden bei Auslegungsschwierigkeiten lässt sich vorher nämlich nicht sagen, welcher Partei das Recht zugesprochen wird; es gilt also, bereits bei der Erstellung des Vertrags dieses Risiko durch klare, verständliche Formulierungen und gegebenenfalls weitere Erläuterungen in der Präambel zu minimieren (siehe hierzu auch die Ausführungen in Abschn. 3.3). 
$\mathrm{Zu}$ diesen Ansprüchen gehören auch Ansprüche auf Schadensersatz. Diese ergeben sich entweder direkt aus Spezialgesetzen (wie beispielsweise dem GeschGehG) oder aus allgemeinen zivilrechtlichen Regelungen bei Verletzung einer Pflicht aus dem Vertrag ( $\$ 280$ ff. BGB). Die Ansprüche bestehen unabhängig davon, ob sie im Vertrag normiert sind oder nicht. Eine vertragliche Wiedergabe kann aber geeignet sein, wenn es aus Sicht der Parteien erforderlich scheint, die bestehenden Konsequenzen und ihre Voraussetzungen klarstellend aufzuzeigen.

Die gesetzlichen Schadensersatzansprüche reichen jedoch nicht in allen Fällen aus, um die eigenen Interessen umfassend zu schützen. Denn zum einen greift eine Schadensersatzpflicht nur bei sog. schuldhaftem Verhalten, d.h., es muss in jedem Fall mindestens Fahrlässigkeit vorliegen. Bei aktivem regelwidrigem Tun wird dies meist der Fall sein, bei schlichter Nichtleistung aber nicht immer. Zum anderen muss ein Schaden natürlich auch beziffert werden können - auch dies ist nicht immer leicht nachzuweisen. Hier können Vertragsstrafen ein geeignetes Mittel sein, da der Anspruch auf die vereinbarte Summe bereits bei festgestelltem Vertragsbruch greift, ohne dass es auf das Vorliegen eines Schadens oder auf ein schuldhaftes Verhalten ankommt. Dadurch wirken Vertragsstrafen auch zugleich als Druckmittel zur Einhaltung des Vertrages.

Die hier genannten Ansprüche verjähren nach drei Jahren ( $\$ 195$ BGB). Es ist möglich, diese Frist durch Vereinbarung angemessen (zu den Grenzen s. 3.3) zu verlängern und dadurch mehr Zeit für eine vielleicht erforderliche Rechtsverfolgung zu schaffen.

Vereinbarungen zu Gerichtsstand oder Schiedsgerichtsbarkeit bieten Vorteile wie beispielsweise ein höheres Maß an Vertraulichkeit, ein schnelleres, flexibleres Verfahren unter Beteiligung von mit der Materie vertrauten Experten (Schiedsgerichtsbarkeit) oder eine Zeit- und Kostenersparnis durch Verkürzung der Anfahrtswege (Gerichtsstand). Sie bergen allerdings auch viele Risiken, was die Prüfung ihrer Zulässigkeit im Einzelfall, ihre rechtssichere Formulierung und die richtige Wahl zwischen verschiedenen Ausgestaltungsmöglichkeiten angeht. Von einer vertraglichen Regelung ohne vorige Hinzuziehung von Experten, die die Umstände des Einzelfalls prüfen, wird daher abgeraten.

\subsection{Prüfpunkte}

Einhaltung der Datenschutzvorgaben bei personenbezogenen Daten. Zwar spielen die Anforderungen der Datenschutzgrundverordnung (DSGVO) und weiterer Schutzgesetze meist keine Rolle, wenn es um Maschinendaten geht. Da jedoch nicht auszuschließen ist, dass in Einzelfällen Maschinendaten Rückschlüsse auf die Person zulassen, die die Maschine bedient, ist an dieser Stelle ein kurzer Exkurs in das Datenschutzrecht geboten. Personenbezogene Daten sind gemäß Art. 4 Nr. 1 DSGVO ,alle Informationen, die sich auf eine identifizierte oder identifizierbare natürliche Person beziehen“. Ist dies gegeben, müssen die Vorgaben der DSGVO beachtet werden, die eine Verarbeitung (darunter fällt auch die Erhebung als solche, Art. $4 \mathrm{Nr}$. 2 DSGVO) nur unter bestimmten Voraussetzungen legitimieren. Vorliegend kommt eine Legitimation in Betracht, etwa durch Einwilligung oder durch eine Kollektivvereinbarung (wie eine Betriebsvereinbarung), wobei letztere Alternative vorzugswürdig 
ist, da sie gegenüber der Einwilligung eine vergleichsweise sichere Rechtsgrundlage bietet und, sofern ein Betriebsrat im Unternehmen besteht, in der vorliegenden Konstellation in den meisten Fällen sowieso abgeschlossen werden muss, um die betriebliche Mitbestimmung gemäß $\S 87$ I Nr. 6 BetrVG zu wahren.(vgl. hierzu im Detail Vogel/Klaus 2019)

Diese Anforderungen treffen zuallererst den Nutzer der Maschine in seiner Eigenschaft als Arbeitgeber und haben für den vorliegend in Rede stehenden Vertrag mit einem Dritten nur dann eine Konsequenz, wenn auch für den Dritten (hier die $\mathrm{H}$ ) personenbezogene Daten vorliegen - denn dann verarbeitet diese ebenfalls personenbezogene Daten gemäß Art. 4 DSGVO (Schwartmann 2018). Wenn es den Parteien also nicht möglich ist, durch technische oder sonstige Vorkehrungen, die für den Dritten nutzbaren Daten so weit zu anonymisieren, dass $\mathrm{H}$ auch nicht mehr theoretisch in der Lage wäre, die betroffenen Personen zu identifizieren, müssen beide Vertragspartner eine Vereinbarung über die Datenverarbeitung abschließen. Diese richtet sich entweder nach Art. 26 DSGVO (bei gemeinsamer Verantwortlichkeit) oder nach Art. 28 DSGVO (Auftragsverarbeitung). ${ }^{11}$ Für beide Konstellationen werden online, beispielsweise von Landesdatenschutzbeauftragten oder Gesellschaften wie der BITKOM, diverse Praxishilfen und Mustervorlagen zur Verfügung gestellt. ${ }^{12}$

(Sonstiges) zwingendes Recht. Es existieren auch bei grundsätzlich bestehendem Gestaltungsspielraum gesetzliche Grenzen, die nicht überschritten werden dürfen. Da sich die Vertragsfreiheit aus der grundrechtlich geschützten allgemeinen Handlungsfreiheit (Art. 2 Abs. 1 GG) ableitet, werden diese Grenzen aus einem dem Allgemeinwohl dienenden Grund beziehungsweise zum Schutz typischerweise unterlegener Vertragsparteien festgelegt ${ }^{13}$ oder sind (wie $§ 138$ BGB, siehe im nächsten Absatz) vergleichsweise hoch angesetzt, um nur krasse Überschreitungen der gegebenen Möglichkeiten abzufangen. Die die hier besprochene Thematik betreffenden Einschränkungen sind daher zum einen überschaubar und betreffen zum anderen Fälle, in denen die Grenzen und Verbote nachvollziehbar sind - die Gefahr, dass es in der vorliegenden Konstellation zu unbeabsichtigten Gesetzesverstößen kommt, ist daher nicht besonders groß.

11 Für weitere Ausführungen siehe beispielsweise Kremer 2018a, b.

12 Siehe zum Beispiel unter https://www.gdd.de/aktuelles/startseite/neue-gdd-praxishilfe-zurgemeinsamen-verantwortlichkeit-nach-art-26-ds-gvo-joint-controllership-abrufbar (letzter Zugriff am 21.07.2021); https://www.frankfurt-main.ihk.de/recht/themen/datenschutzrecht/muster_auftragsverarbeitung/ (letzter Zugriff am 21.07.2021); https://www.badenwuerttemberg.datenschutz.de/wp-content/uploads/2018/01/muster_adv.pdf (letzter Zugriff am 21.07.2021).

13 Ein sog. Kontrahierungszwang findet sich beispielsweise in den Bereichen der Daseinsvorsorge: Kein Supermarkt kann den Abschluss eines Kaufvertrages mit einer Person verweigern, kein Unternehmen des öffentlichen Personenverkehrs den Abschluss eines Beförderungsvertrags. Viele Vorgaben gibt es etwa auch im Miet- oder Arbeitsrecht. 
Das gilt etwa für $§ 138$ BGB, wonach sittenwidrige Rechtsgeschäfte nichtig sind. Die Norm selbst enthält in ihrem Absatz 2 schon eine Erläuterung, welche Rechtsgeschäfte dies ,insbesondere“ sein können, nämlich „Rechtsgeschäfte, durch die jemand unter Ausbeutung der Zwangslage, der Unerfahrenheit, des Mangels an Urteilsvermögen oder der erheblichen Willensschwäche eines anderen sich oder einem Dritten für eine Leistung Vermögensvorteile versprechen oder gewähren lässt, die in einem auffälligen Missverhältnis zu der Leistung stehen.“ Da die Wahrscheinlichkeit, aus Versehen ein sittenwidriges Rechtsgeschäft abzuschließen, äußerst gering ist, wird auf weitere Ausführungen an dieser Stelle verzichtet.

Nichtig ist gemäß $\S 18,19$ GWB i.V. m. $§ 134$ BGB ein Rechtsgeschäft, welches unter Missbrauch der marktbeherrschenden Stellung erfolgt. Die Beurteilung, ob eine marktbeherrschende Stellung vorliegt, richtet sich nach den umfangreichen Kriterien und Auslegungshilfen des $\S 18$ GWB, wonach unter anderem der Zugang zu wettbewerbsrelevanten Daten bei dieser Beurteilung Berücksichtigung finden soll $(\S 18$ Abs. 3a Nr. 4 GWB). Nach herrschender Ansicht ist die Rechtsfolge nach Sinn und Zweck der Norm jedoch dergestalt zu reduzieren, dass das entsprechende Rechtsgeschäft nicht komplett nichtig, sondern ein Vertrag zu den hiernach noch zulässigen Konditionen fortbesteht (Armbrüster 2018).

Eine weitere, hier relevante, Grenze enthält $\S 202$ BGB für Abreden zur Verjährungsfrist. Hiernach sind Verkürzungen der Frist, wenn sie sich auf einen Anspruch aus Haftung wegen Vorsatzes beziehen und Verlängerungen, die 30 Jahre überschreiten, nicht möglich. Eine solche Klausel wäre unwirksam, sodass an ihre Stelle die gesetzliche Verjährungsfrist von drei Jahren treten würde (Grothe 2018).

$\mathrm{Zu}$ beachten sind ferner die Vorschriften der AGB-Kontrolle ( $\$ \$ 305 \mathrm{ff}$. BGB), die strengere Maßstäbe an die Wirksamkeit einzelner Klauseln anlegen als das ,normale“ Schuldrecht und die greifen, wenn eine Partei der anderen bei Vertragsschluss „für eine Vielzahl von Verträgen vorformulierte Vertragsbedingungen“ stellt (§ 305 Abs. 1 BGB). Hierbei ist gleichgültig, ob die Bestimmungen einen äußerlich gesonderten Bestandteil des Vertrags bilden oder in die Vertragsurkunde selbst aufgenommen werden oder welchen Umfang sie haben. AGB in diesem Sinne liegen nur dann nicht vor, wenn die Bedingungen im Einzelnen ausgehandelt sind. Anwendung finden die $\S \S 305$ ff. BGB (in eingeschränktem Umfang) auch bei Rechtsgeschäften zwischen zwei Unternehmern, ( $\$ 310$ Abs. 1 Satz 1 BGB). Allerdings beinhalten hier alle in Betracht kommenden Verbote einen Wertungsspielraum, indem sie die Unwirksamkeit von Erfordernissen wie etwa „Unangemessenheit“ und „Unzumutbarkeit“ abhängig machen. Hier können unter anderem folgende Fallgruppen relevant werden:

- Bei der Festlegung von Abwicklungszahlen bei Vertragsbeendigung können die Bestimmungen des $\S 308$ Abs. 1 Nr. 7 BGB, für AGB gegenüber Unternehmern über $\$ 307 \mathrm{BGB}$, relevant werden.

- Die Vereinbarung von Vertragsstrafen in AGB für bestimmte Leistungsverstöße: Diese sind gegenüber Verbrauchern fast immer unwirksam gemäß § 309 Nr. 6 BGB. Eine Vereinbarung unter Unternehmern muss sich ,nur' an $\S 307$ BGB und damit im Ergebnis daran messen lassen, ob sie den Vertragspartner entgegen Treu und Glauben unangemessen benachteiligen.

- Für Haftungsausschlüsse gelten insbesondere die Bestimmungen des $§ 309$ Nr. 7 und 8 BGB. 
Da im vorliegenden Beitrag im Einzelnen ausgehandelte Vertragswerke besprochen werden, erfolgt an dieser Stelle keine umfassende Einordnung der genannten Beispiele. Wichtig ist, sich darüber bewusst zu sein, dass bei Verwendung von AGB strengere Grenzen gelten können, und diesbezüglich entsprechend aufmerksam zu sein.

Klarheit und Bestimmtheit der getroffenen Vereinbarung. Der Vertrag sollte vor Abschluss dahingehend überprüft werden, ob die Regelungen die gewünschten Folgen korrekt wiedergeben und präzise formuliert worden ist. Insbesondere bei Bestimmungen, die Rechte und Pflichten normieren, sollten die Sätze so kurz wie möglich sein. Auf Erläuterungen oder verschachtelte Aussagen ist im Vertrag selbst zu verzichten (hierfür kann und sollte die Präambel genutzt werden, s. o.). Auch sind mehrdeutige oder widersprüchliche Formulierungen zu vermeiden. Die Aussagen müssen sich im Ergebnis daran messen lassen, ob auch ein an den Verhandlungen nicht beteiligter Dritter ohne weitere Erläuterung einen Sachverhalt mit ihnen lösen kann.

\section{Fazit und Checkliste}

Die folgende Checkliste (Tab. 1) fasst die genannten Punkte (im Vertrag sichtbar oder nicht) zusammen und soll ein Grundgerüst dafür bieten, einen ähnlich gelagerten Sachverhalt hieran orientierend vertraglich $\mathrm{zu}$ regeln, wobei die Vertragsparteien die jeweiligen Bestimmungen anhand der Umstände und Interessen des Einzelfalls, der Art der Geschäftsbeziehung und der technischen Machbarkeit wählen und interessengerecht formulieren können. Dabei kann es erforderlich werden, das Knowhow unterschiedlicher Disziplinen einzubeziehen, neben Personen mit technischem Sachverstand beispielsweise auch solche mit juristischem oder ökonomischem Hintergrund. Hier ist es notwendig, eine gemeinsame Sprache zu finden, was Herausforderung und Aufforderung zugleich ist. Je technischer und für den Laien abstrakter die Bedingungen sind, desto mehr Verständnis für die Bedürfnisse der anderen Seite ist gefragt. Geerenstein schreibt hierzu treffend: „Juristen müssen sich zwangsläufig mit technischen Sachverhalten beschäftigen, andersherum ist es aber auch essenziell, dass die andere Seite eine gewisse Sensibilität für die Bedürfnisse der Vertragsgestaltenden entwickelt und klar wird, dass kleinste Unklarheiten oder Abweichungen $\mathrm{zu}$ einer völlig anderen Bewertung und damit $\mathrm{zu}$ einer notwendig anderen Ausgestaltung führen können“ (Geerenstein 2020). 
Tab. 1. Die Klammerzusätze zeigen an, ob ein Punkt seinen Niederschlag im Vertrag findet (§) oder lediglich als Prüfpunkt (PP) in die Überlegungen mit einbezogen werden muss

\begin{tabular}{l|l}
\hline 1 & Definitionen, Erläuterungen und Hintergründe (Präambel) \\
\hline 2 & Vertragsparteien $(\S)$ \\
\hline 3 & Vertragsgegenstand (§) \\
\hline 5 & Leistungspflichten, Zugriffsrechte und Nutzungszwecke $(\S)$ \\
\hline 6 & Vereinbarungen über die Beschaffenheit des Vertragsgegenstands (§) \\
\hline 7 & $\begin{array}{l}\text { Lestimmungen technischer Art zur Übertragung der Daten }(\S) \\
\text { gegenstand bei Vertragsende) }(\S)\end{array}$ \\
\hline 8 & $\begin{array}{l}\text { Vertragliche Vereinbarungen über die Haftung für durch fehlerhafte Daten entstandene } \\
\text { Schäden (§) }\end{array}$ \\
\hline 9 & $\begin{array}{l}\text { Fragen der Vertragsdurchsetzung (§) } \\
\bullet \text { Hinweis auf gesetzliche oder vertraglich modifizierte Rechtsfolgen } \\
\bullet \text { Vertragsstrafen } \\
\bullet \text { Verjährung } \\
\bullet \text { Vereinbarungen zu Gerichtsstand und Schiedsgerichtsbarkeit }\end{array}$ \\
\hline 10 & Einhaltung der Datenschutzvorgaben bei personenbezogenen Daten (PP/§) \\
\hline 11 & Beachtung zwingenden Rechts (PP) \\
\hline 12 & Prüfung von Klarheit und Widerspruchsfreiheit der Formulierungen (PP) \\
\hline
\end{tabular}

\section{Literatur}

Apel, S.: Datenkaufvertrag. In: Nägele, T., Apel, S. (Hrsg.) Beck'sche Online-Formulare ITund Datenrecht, 7. Aufl., Stand 01.05.2021. Beck, München (2021)

Armbrüster, C.: BGB § 134 - Gesetzliches Verbot. In: Säcker, F.J., Rixecker, R., Oetker, H., Limperg, B. (Hrsg.) Münchener Kommentar zum Bürgerlichen Gesetzbuch Band 1. Beck, München (2018)

Bundesverfassungsgericht (BVerfGE), Urteil vom 15. Dezember 1983 - 1 BvR 209/83

Emmerich, V.: BGB $§ 311$ - Rechtsgeschäftliche und rechtsgeschäftsähnliche Schuldverhältnisse. In: Säcker, F.J., Rixecker, R., Oetker, H., Limperg, B. (Hrsg.) Münchener Kommentar zum Bürgerlichen Gesetzbuch Band 3. Beck, München (2019)

Europäische Kommission, Vorschlag für eine Verordnung des Europäischen Parlaments und des Rates über einen Rahmen für den freien Verkehr nicht personenbezogener Daten in der Europäischen Union vom 13.09.2017 - 2017/0228 (COD)

Froese, J., Straub, S.: Wem gehören die Daten? - Rechtliche Aspekte der digitalen Souveränität in der Wirtschaft. In: Hartmann, E.A. (Hrsg.) Digitalisierung souverän gestalten Innovative Impulse im Maschinenbau, S. 86-97. Springer Vieweg, Berlin (2020)

Gaier, R.: BGB $\S 314$ - Kündigung von Dauerschuldverhältnissen aus wichtigem Grund. In: Säcker, F.J., Rixecker, R., Oetker, H., Limperg, B. (Hrsg.) Münchener Kommentar zum Bürgerlichen Gesetzbuch Band 3. Beck, München (2019)

Geerenstein, D.: Maschinenbau und Industrie 4.0. In: Frenz, W. (Hrsg.) Handbuch Industrie 4.0.: Recht, Technik, Gesellschaft, S. 637-258. Springer, Berlin (2020)

Grothe, H.: BGB § 202 - Unzulässigkeit von Vereinbarungen über die Verjährung. In: Säcker, F.J., Rixecker, R., Oetker, H., Limperg, B. (Hrsg.) Münchener Kommentar zum Bürgerlichen Gesetzbuch Band 1. Beck, München (2018) 
Hoeren, T., Uphues, S.: Big Data in Industrie 4.0. In: Frenz, W. (Hrsg.) Handbuch Industrie 4.0.: Recht, Technik, Gesellschaft, S. 113-131. Springer, Berlin (2020)

Kremer, S.: DS-GVO Art. 26 - Gemeinsam für die Verarbeitung Verantwortliche. In: Schwartmann, R., Jaspers, A., Thüsing, G., Kugelmann, D. (Hrsg.) Heidelberger Kommentar DS-GVO/BDSG. C.F. Müller, Heidelberg (2018)

Kremer, S.: DS-GVO Art. 28 - Auftragsverarbeiter. In: Schwartmann, R., Jaspers, A., Thüsing, G., Kugelmann, D. (Hrsg.) Heidelberger Kommentar DS-GVO/BDSG. C.F. Müller, Heidelberg (2018)

Kuß, C.: Vertragstypen und Herausforderungen für die Vertragsgestaltung. In: Sassenberg, T., Faber, T. (Hrsg.) Rechtshandbuch Industrie 4.0 und Internet of Things, S. 387-433. Beck, München (2020).

Sattler, A.: Schutz von maschinengenerierten Daten. In: Sassenberg, T., Faber, T. (Hrsg.) Rechtshandbuch Industrie 4.0 und Internet of Things, S. 35-75. Beck, München (2020)

Schwartmann, R.: DS-GVO Art. 4 - Begriffsbestimmungen. In: Schwartmann, R., Jaspers, A., Thüsing, G., Kugelmann, D. (Hrsg.) Heidelberger Kommentar DS-GVO/BDSG. C.F. Müller, Heidelberg (2018)

Vogel, P., Klaus, A.: Zulässigkeit der Verarbeitung von GPS-Daten im Arbeitsverhältnis. In: Stich, V., Schumann, J., Beverungen, D., Gudergan, G., Jussen, P. (Hrsg.) Digitale Dienstleistungsinnovationen, S. 393-496. Springer, Heidelberg (2019)

Vogel, P.: Datenhoheit in der Landwirtschaft 4.0. In: Gansdorfer, M. et al. (Hrsg.) Digitalisierung für Mensch, Umwelt und Tier. Referate der 40. GIL-Jahrestagung. Bd. Gesellschaft für Informatik, Bonn (2020)

Wiebe, A.: Von Datenrechten und Datenzugang - Ein rechtlicher Rahmen für die europäische Datenwirtschaft. Comput. Recht, 87-93 (2017)

Open Access Dieses Kapitel wird unter der Creative Commons Namensnennung 4.0 International Lizenz (http://creativecommons.org/licenses/by/4.0/deed.de) veröffentlicht, welche die Nutzung, Vervielfältigung, Bearbeitung, Verbreitung und Wiedergabe in jeglichem Medium und Format erlaubt, sofern Sie den/die ursprünglichen Autor(en) und die Quelle ordnungsgemäß nennen, einen Link zur Creative Commons Lizenz beifügen und angeben, ob Änderungen vorgenommen wurden.

Die in diesem Kapitel enthaltenen Bilder und sonstiges Drittmaterial unterliegen ebenfalls der genannten Creative Commons Lizenz, sofern sich aus der Abbildungslegende nichts anderes ergibt. Sofern das betreffende Material nicht unter der genannten Creative Commons Lizenz steht und die betreffende Handlung nicht nach gesetzlichen Vorschriften erlaubt ist, ist für die oben aufgeführten Weiterverwendungen des Materials die Einwilligung des jeweiligen Rechteinhabers einzuholen.

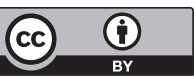

